\title{
Research on Dynamic Replication Routing Protocols for Opportunistic Networks
}

\author{
Jiayang Tian ${ }^{1, a}$, Yifei Sun ${ }^{2, b}$ \\ ${ }^{1}$ International School, Beijing University of Posts and Telecommunications, Beijing 102209, China; \\ ${ }^{2}$ College of Sciences, North China University of Technology, Beijing, 100144, China. \\ atjyjiayutong@163.com, byifeisun1@163.com
}

Keywords: Opportunistic network, routing algorithm, efficiency

\begin{abstract}
Opportunistic network is a self-organization network using the meeting opportunity of nodes movement to achieve network communication. There is no complete path between the source node and the destination node in this kind of network. A lot of routing algorithms are proposed based on this network, which can be roughly classified into two categories, one is based on replication, including epidemic routing, spray\&wait routing and spray\&focus routing, the other is based on retransmission, such as direct-delivery routing, first contact routing and prophet routing. This paper proposes a method to improve the spray\&wait routing algorithm in the opportunistic networks, and examines its performance on the ONE platform to verify that the improved routing algorithm achieves the expected performance compared with other routing algorithms.
\end{abstract}

\section{Introduction}

Opportunistic networks are intermittently connected mobile wireless networks. Most time there is no complete path from the source node to the destination node, or such a complete path is so unstable that it will be changed and broken soon after it has been found [1]. There may not be an end to end path between source and destination in the opportunistic network. A large number of wireless Ad-hoc routing protocols have been proposed in the past. However, the traditional Ad-hoc routing protocols are not applicable for the opportunistic network. Even if the network has only a slight disconnection, the performance of these protocols will become very poor. Because of the difficulty in obtaining the information of the connection and the transmission, the transmission of the message presents a characteristic of opportunistic forwarding [2,3]. When the next hop node to the destination node is not in the routing table, the message is cached on the current node, and as the current node moves waiting for the appropriate forwarding opportunity. For each message determining the best next hop node and selecting the appropriate time for forwarding becoming the key issues in the design of the high efficiency opportunity network routing protocol. The message forwarding of the opportunity network is shown in Figure 1.

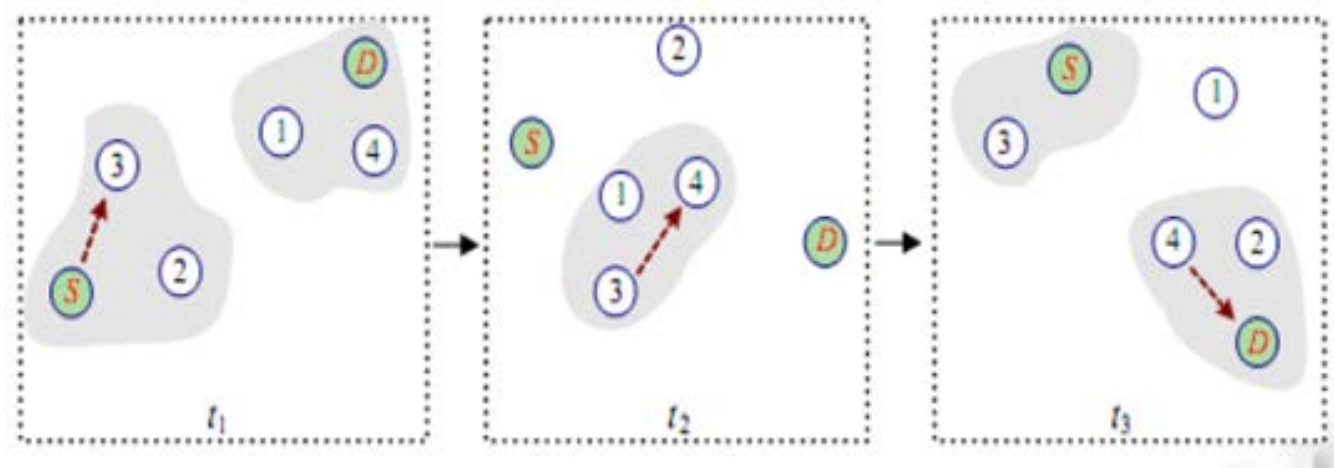

Fig.1 Message forwarding in opportunistic networks

The research object of this paper is to propose a method to improve the spray\&wait routing algorithm in opportunistic networks, and examine its performance on the ONE platform to verify that the improved routing algorithm could achieve the expected performance compared with other routing algorithms. 


\section{Dynamic Replication Combining with Utility Forwarding}

Compared to other replication-based routing protocols, spray\&wait algorithm and spray\&focus algorithm have great advantages. But there are still two problems. Firstly, how to dynamically control the number of copies of messages that can guarantee successful delivery of messages, and not cause unnecessary network overhead [4]. Secondly, how to effectively forward copies of the message, passing the message to the destination with the maximum probability.

Realization of Dynamic Replication. Too less copies of the message in the network can not guarantee the success of the message, but too much copies can lead to the waste of network resources and even cause network congestion [5, 6]. Dynamic replication means that the number of copies of the message in the network is not fixate and change with the situation of the network and the forwarding ability of the node. When two nodes meet each other, we need to decide the number of copies of each node, we can make a simple calculation according to the equation (1).

$$
p_{\text {init }}=1-\left(1-p_{a}\right)^{n_{1}}\left(1-p_{b}\right)^{n_{2}}
$$

where $p_{\text {init }}$ represents the expected success rate of messages forwarding in the entire network, $p_{a}$ represents the probability of node a meeting with destination node, $p_{b}$ represents the probability of node $b$ meeting with destination node. When node a encounters node $b$, it should make a judgment firstly. Assuming that the number of copies of a message in one node is $L$, if $1-\left(1-p_{a}\right)^{L / 2}\left(1-p_{b}\right)^{L / 2}$ is less than $p_{\text {init }}$, indicating that we need to increase the number of copies. Otherwise, indicating that we need to decrease the number of copies. Then Compare $p_{a}$ with $p_{b}$, distributing copies of messages according to the principle of the nodes with large probability meeting with destination node carrying a large number of copies. Assuming $p_{a}>p_{b}$, make a specific description. If you need to increase the number of copies according to the first judgment, then set the number of copies in node $\mathrm{b}$ to $\mathrm{L} / 2$, the number of copies in the node a is increased from $\mathrm{L} / 2$ until equation 1 is satisfied. If you need to decrease the number of copies according to the first judgment, then set the number of copies in node a to $\mathrm{L} / 2$, the number of copies in the node $\mathrm{b}$ is decreased from $\mathrm{L} / 2$ until equation 1 is satisfied.

Through the process above, both guarantee the success probability of the message delivery, and optimize the distribution of copies as much as possible by making nodes with large probability meeting with destination node carrying more copies than nodes with little probability.

Introduction of Utility Mechanism. Message utility is a quantitative value for the importance of a message or an emergency of a message. Make sure the message that with great utility value can be deliveried quickly [7]. Node utility is the ability to transmit messages. The following factors affect the utility of the message significantly:

(1) Remaining life of message. The node having shorter remaining life is more likely to be discarded, and greater priority should be given.Set this kind of nodes with great utility value.

(2) The number of messages be forwarded. Messages which have been forwarded many times is not easy to find destination node, and have wasted some network resources. These messages should leave forwarding chance to those who have just generated or forwarding only a few times.The utility of these messages should be little.

(3) Message size. The messages with small size take little storage space and are easy to forward. Compared to the big news, there should be a greater utility value.

In summary, the utility function can be expressed as a formula 2. "mu" is utility value. "counthops" is the number of messages forwarding times. "messagesize" is message size. "remainttl" is remaining life of message. " $R$ " changes basing on the above three factors in the range of $[0,1]$.

$$
m u=R *(1 / \text { (counthops* messagesize* remainttl)) }
$$

Implementation of Routing Algorithm. This routing protocol is based on the spray\&wait routing protocol. So we named this routing algorithm Newsprayandwait. Specifically, in dispersed phase, the nodes select the message with greatest utility value set which to forward.In other words, forwarding is based on utility values. The order in which messages are transmitted is determined by the utility 
value of themselves. For copies of the message,using dynamic replication method to determine the copies of each message that meeting nodes carried.

\section{Simulation Results}

In this paper, the simulation tool is ONE platform. The simulation platform was developed by University of Helsinki, Finland, and it is a network simulation tool based on java. The simulation parameters are shown in Table 1.

Table 1 Simulation parameters setting

\begin{tabular}{ccccccc}
\hline Groups & Nodes & Speed of nodes & $\begin{array}{c}\text { Cache of } \\
\text { nodes }\end{array}$ & Mobile model & Event generator & Mode of report \\
\hline Bus & 6 & {$\left[\begin{array}{ll}6,8 & 8\end{array}\right.$} & $20 \mathrm{M}$ & Basing on path & Message generator & MessageStatsReport \\
Car & 6 & {$\left[\begin{array}{ll}7,10\end{array}\right]$} & $10 \mathrm{M}$ & Basing on path & Message generator & MessageStatsReport \\
pedestrian & 60 & {$\left[\begin{array}{ll}1,3 & 3\end{array}\right.$} & $5 \mathrm{M}$ & Basing on map & Message generator & MessageStatsReport \\
\hline
\end{tabular}

This paper simulates four replication-based routing protocols, including epidemic, spray \&wait, spray\&focus and newsprayandwait. The performance of these routing protocols is studied by comparing 3 performance indicators including successful rate of data transmission, network overhead, and average delay. The simulation results are shown in Figure 2, Figure 3 and Figure 4.

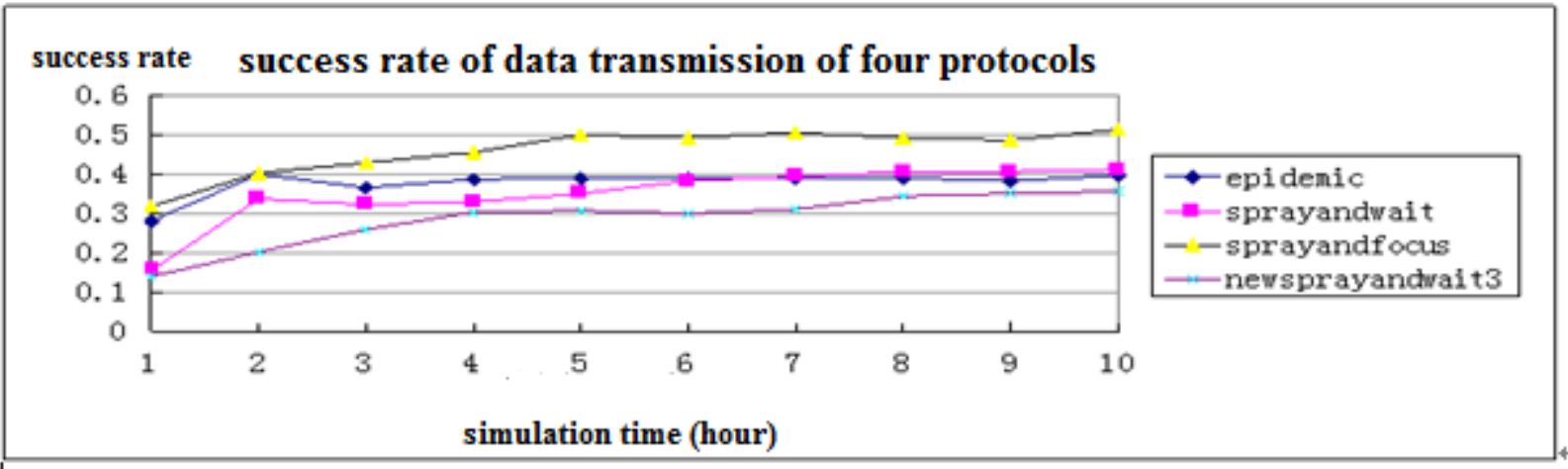

Fig.2 Successful rate of data transmission of four protocols

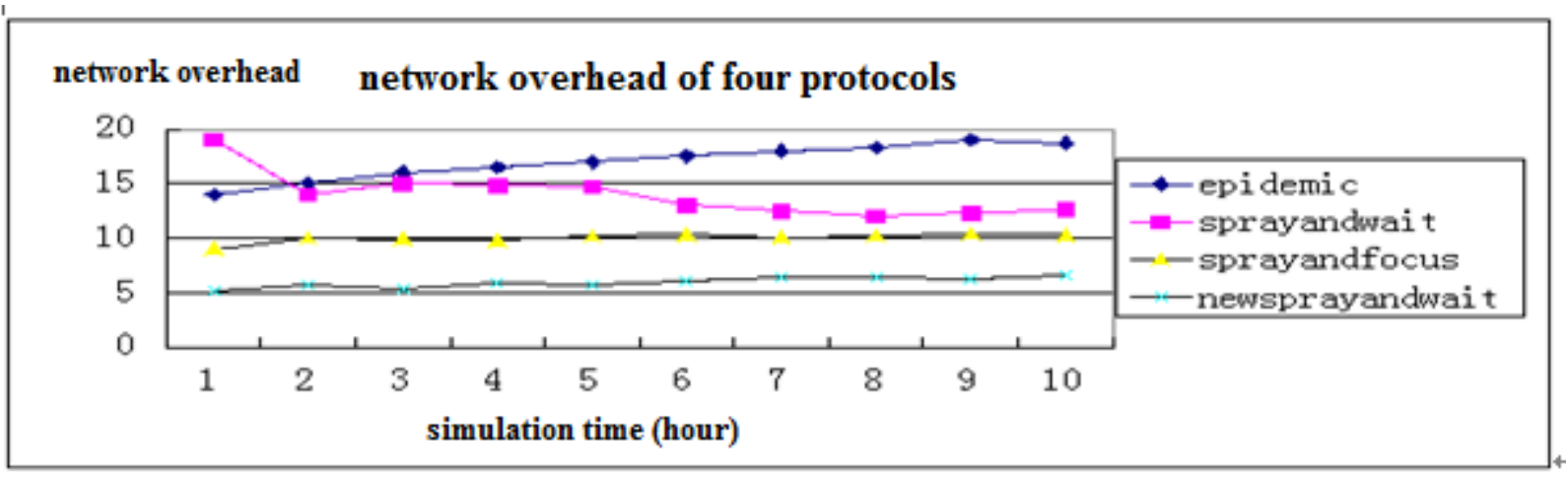

Fig.3 Network overhead of four protocols 


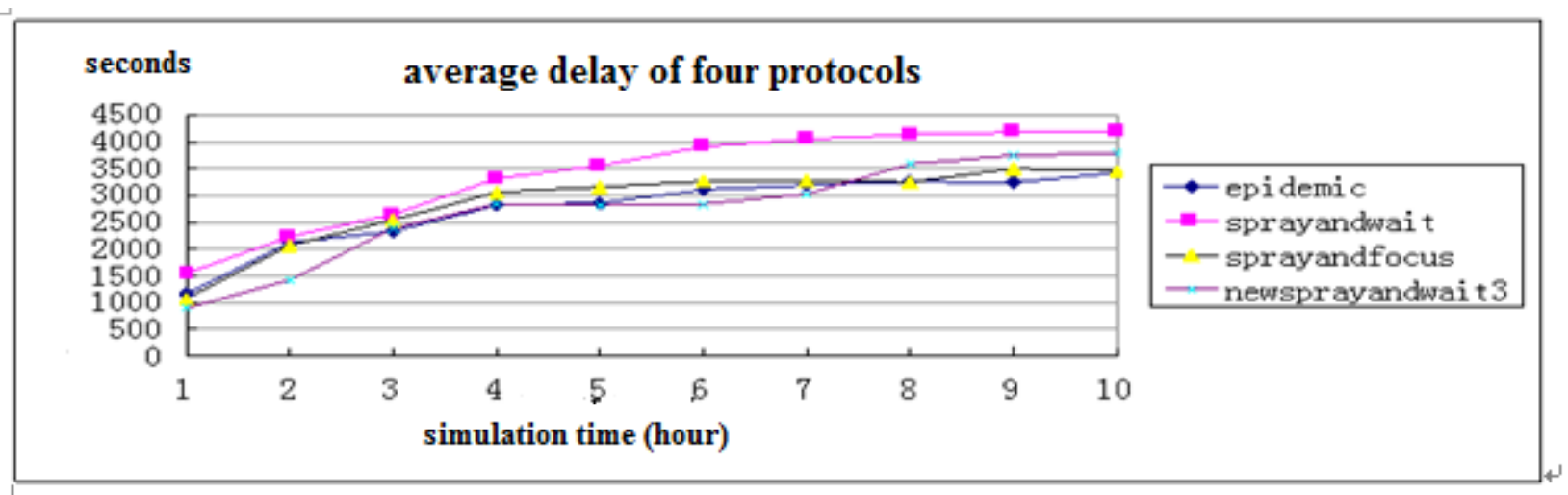

Fig.4 Average delay of four protocols

According to the simulation results above, when the simulation time is long enough, the average delay time of these four routing protocols has little difference. However, in terms of network overhead rate and data transmission rate, different routing protocols have different characteristics. As clearly seen from the table, epidemic routing algorithm has the highest data success rate and highest network overhead rate. When it comes to transmission rate, spray \&wait is almost equivalent to spray \& focus, but the network overhead rate of spray\&focus is relatively low. The new proposed algorithm newsprayandwait has poor performance in terms of data transmission efficiency, but it has the best performance in terms of network overhead.

\section{Conclusion}

This paper analyzes and compares several routing algorithms based on replication, and improves the shortcomings of the existing routing algorithms. That is, based on the spray\&wait routing algorithm, introducing the dynamic control mechanism and utility mechanism of messages, so as to realize the dynamic copying of messages and improve the success rate of forwarding. Finally, the algorithm is implemented on the ONE platform, and the simulation experiment is carried out. Through research and experiment, several laws are found. The routing protocols with high data transmission tend to rely on a large number of copies of messages, but also bring a lot of network redundancy so the consequence is the great increaing of network overhead rate. In the opportunistic network, the so-called excellent routing algorithm has to make a balance between the success rate of data transmission and the network efficiency.

\section{References}

[1]Akyildiz IF, Akan B, Chen C, Fang J, Su W. InterPlaNetary Internet: State-of-the-Art and research challenges. Computer Networks.2003.43(2):75-112.

[2] Musolesi M, Mascolo C. CAR: Context-Aware adaptive routing for delay tolerant mobile networks. IEEE Trans. on Mobile Computing. 2009, 8(2):246-260.

[3] Pentland A, Fletcher R, Hasson A. DakNet: Rethinking connectivity in developing nations. Computer Networks. 2004.37(1):78-83.

[4] Ari Keränen, Jörg Ott, Teemu Kärkkäinen. The ONE simulator for DTN protocol evaluation. SimuTools.2009: 55

[5] Pei-Yan Yuan, Hua-Dong Ma, Peng-Rui Duan. Impact of Strangers on Opportunistic Routing Performance[J]. Journal of Computer Science and Technology . 2013 (3).

[6] Qinghua Li, Wei Gao, Sencun Zhu, Guohong Cao. A routing protocol for socially selfish delay tolerant networks[J]. Ad Hoc Networks. 2011 (8).

[7] Bhorkar A A, Naghshvar M, Javidi T, et al. Adaptive opportunistic routing for wireless ad hoc networks[J]. IEEE/ACM Transactions on Networking (TON), 2012, 20(1): 243-256. 